\title{
CORRECTION
}

View Article Online

View Journal | View Issue

Check for updates

Cite this: Mater. Chem. Front., 2021, 5, 7684

DOI: $10.1039 /$ d1qm90074a

rsc.li/frontiers-materials

\section{Correction: Poly(3,4-ethylenedioxythiophene) bearing fluoro-containing phenylboronic acid for specific recognition of glucose}

\author{
Wenji Bao, ${ }^{a}$ Wenfeng Hai, ${ }^{a}{ }^{a}$ Layue Bao, ${ }^{a}$ Fan Yang, ${ }^{a}$ Yushuang Liu, ${ }^{* a}$ Tatsuro Goda ${ }^{b}$ \\ and Jinghai Liu*a \\ Correction for 'Poly(3,4-ethylenedioxythiophene) bearing fluoro-containing phenylboronic acid for \\ specific recognition of glucose' by Wenji Bao et al., Mater. Chem. Front., 2021, DOI: 10.1039/ \\ d1qm00926e.
}

The authors regret that the funding acknowledgements were omitted from the manuscript. The acknowledgements are as follows.

The work was financially supported by the National Natural Science Foundation of China (22164016, 22104066, 21961024), the Inner Mongolia Natural Science Foundation (2020BS02007, 2020BS08007), Inner Mongolia Autonomous Region Science \& Technology Planning Project for Applied Technology Research and Development (2019GG261), the Research Program of Science and Technology at Universities of Inner Mongolia Autonomous Region (NJZY20117), the State Key Laboratory of Applied Optics (SKLAO-201911), the Doctoral Scientific Research Foundation of Inner Mongolia University for Nationalities (Project No.: BS453, BS515).

The Royal Society of Chemistry apologises for these errors and any consequent inconvenience to authors and readers.

\footnotetext{
${ }^{a}$ Inner Mongolia Key Laboratory of Carbon Nanomaterials, Nano Innovation Institute (NII), College of Chemistry and Chemical Engineering, Inner Mongolia Minzu University, Tongliao 028000,China. E-mail: hai_wenfeng@163.com, yushuangliu1989@163.com, jhliu2008@sinano.ac.cn

${ }^{b}$ Department of Biomedical Engineering, Faculty of Science and Engineering, Toyo University, 2100 Kujirai, Kawagoe, 350-8585 Saitama, Japan
} 